\title{
Les « travaux personnels encadrés » au lycée
}

Ambitions et modestie

Tutoring initiatives in secondary schools - a tentative step forward

Los "trabajos personales dirigidos" en el instituto. Ambiciones y modestias

\section{Marc Baconnet}

\section{OpenEdition}

\section{Journals}

Édition électronique

URL : https://journals.openedition.org/ries/1844

DOI : 10.4000/ries. 1844

ISSN : 2261-4265

\section{Éditeur}

France Education international

Édition imprimée

Date de publication : 1 avril 2002

Pagination : 47-51

ISBN : 2-84520-553-8

ISSN : $1254-4590$

Référence électronique

Marc Baconnet, "Les «travaux personnels encadrés » au lycée », Revue internationale d'éducation de Sèvres [En ligne], 29 | avril 2002, mis en ligne le 01 avril 2005, consulté le 05 juillet 2021. URL : http:// journals.openedition.org/ries/1844; DOI : https://doi.org/10.4000/ries.1844 


\section{Les « travaux personnels encadrés " au lycée}

\section{Ambitions et modestie}

\section{Marc Baconnet}

Pour qu'un programme, et encore plus une innovation, aient quelque chance d'être compris et bien reçus, il faut que les concepteurs et, après eux, le législateur remplissent au moins trois conditions :

- qu'il y ait un minimum de cohérence avec les pratiques ou les évolutions que l'on constate autour de ce nouveau programme, en amont et en aval;

- qu'il réponde à d'évidents besoins et à des urgences incontestables ;

- qu'il ne se refuse pas la possibilité de prendre en compte, pour mieux les accompagner, ce que les pratiques des enseignants ont parfois, ou souvent, anticipé en marge des textes officiels.

Ces trois conditions me semblent avoir été réunies dans la conception et la définition des Travaux personnels encadrés, dénommés plus brièvement TPE.

En effet ce serait une erreur de croire que les TPE ont été improvisés et décrétés par une volonté réformatrice brouillonne. Ils s'inscrivent dans la continuité de ce qui se cherche, et se fait, au collège avec les itinéraires de découverte, et de ce qui se pratiquera plus tard dans la poursuite des études, avec par exemple les TIPE des classes préparatoires, et plus généralement les habitudes de travail de l'enseignement supérieur.

On pourra ironiser sur les changements rapides et quelques peu démobilisateurs intervenus ces dernières années en collège : parcours diversifiés, travaux croisés, itinéraires de découverte. Tâtonnements irritants, qui donnent l'impression d'une institution qui hésite, se trompe et s'essouffle. Certes, mais elle a en tout cas le mérite d'avouer ses hésitations, d'apporter très vite des correctifs à des solutions imparfaites face à des questions auxquelles il faut répondre. Et c'est bien la même logique qui préside aux itinéraires de découverte et aux TPE. Il s'agit de répondre à deux questions qui se posent d'ellesmêmes :

- Comment aujourd'hui un élève de collège ou de lycée peut-il apprendre à accéder de la manière la plus autonome possible au savoir (entendu ici en un sens très large) ?

- Comment peut-il prendre progressivement conscience que ce savoir est un perpétuel tissage qui implique aussi une mise en rapport avec autre chose que le cadre étroitement délimité de son objet d'étude et de la discipline dans laquelle il travaille? 
Pour répondre à ces deux demandes, pour ne pas dire à ces deux urgences, il convient de viser deux objectifs, l'un très concret, l'autre de nature plus épistémologique :

- apprendre à se servir des moyens d'information et de communication tels qu'ils fonctionnent aujourd'hui ;

- découvrir progressivement que les éléments du savoir ne se réduisent pas à l'étiquette d'un exercice, d'une rubrique ou d'une discipline.

Rappelons que les TPE concernent les classes de première et terminales. «En première comme en terminale, les élèves auront à mener un seul TPE qui croisera deux ou trois disciplines [...]. À chacun des deux niveaux, soixantedouze heures par division seront à répartir entre les professeurs concernés... ${ }^{1}$ Le sujet est arrêté après concertation et accord entre les enseignants et leurs élèves, dans le cadre proposé par une liste nationale.

Un des buts recherchés par ce type d'activité est donc d'intégrer dans les objectifs de l'enseignement les conditions nouvelles de l'accès au savoir et de sa transmission. Refuser une telle opportunité serait commettre une grave erreur. Or cela n'est pas simple. Les élèves, mais aussi leur professeur et tout individu qui vit dans le monde aujourd'hui, risquent fort d'être dépassés et submergés par la multiplicité et la diversité des supports. Le risque est grand de recevoir l'information de façon passive et anarchique. Il faut apprendre à choisir, à utiliser, à critiquer, pour pouvoir mettre en rapport ce qui est trouvé avec le sujet qu'on doit traiter. Il faut, au sens précis de l'expression, s'y retrouver, et non pas s'y perdre. C'est malheureusement plus souvent ce risquelà qui l'emporte. Dans le monde contemporain tout y pousse, et de façon parfois d'autant plus perverse que l'information est "médiatisée », toute prête à être consommée, sans même qu'on en perçoive le sens et la portée.

D'autre part, en ayant obligation d'utiliser les ressources d'au moins deux disciplines, on cherche à faire prendre conscience que le savoir est un tissage de différentes données et disciplines qui contribuent au développement des sciences et des techniques. Toutes les disciplines évoluent, non seulement dans leurs contenus, mais aussi dans leur méthodologie, et beaucoup plus vite que ne peut le laisser voir la répétition des mêmes connaissances et des mêmes méthodes dans un enseignement qui a en effet besoin de repères stables pour pouvoir se transmettre. C'est là une contradiction inhérente à l'enseignement qu'on souligne rarement. On ne peut enseigner que dans des cadres fixes, et on doit enseigner un savoir qui évolue, ou en tout cas préparer les esprits à admettre cette évolution. Les TPE offrent de ce point de vue une très heureuse ouverture : c'est le moment de se confronter à une information vivante, qui se fait et rend «actuelle », quel que soit le sujet proposé, la recherche. 
L'objectif essentiel est d'apprendre à accéder de la manière la plus autonome possible au savoir. Le travail autonome, les modules, l'aide individualisée avaient aussi cette visée, mais on restait plus ou moins dans le cadre de la classe. Ici on s'en sépare plus nettement, et l'ambition est très forte. À tel point que dès le départ de regrettables malentendus se sont instaurés. On a parfois cru que les TPE étaient une initiation à la recherche, sans trop préciser de quelle recherche il peut s'agir, et malheureusement certains thèmes proposés aux élèves ressemblent à des sujets de thèses universitaires. Pour ne vexer personne on nous pardonnera de ne donner aucun exemple. Une fois de plus on a mesuré combien la projection sans nuance des modèles et des pratiques universitaires dans l'enseignement secondaire est instinctive, et nocive. Pourtant l'objectif est très clair, ne serait-ce que par la force des choses. Il ne peut s'agir de recherche au sens universitaire du terme. Le terme du reste ne convient pas. S'il s'agit de recherche, elle ne peut être essentiellement que de d'information et de documentation, elle ne peut conduire qu'aux portes de la véritable recherche, c'est-à-dire aux saines interrogations d'un jeune esprit qui prend conscience par lui-même que les choses sont plus complexes qu'il n'y paraît. Le but est d'apprendre à se servir de l'information et de la documentation recueillies pour produire une synthèse claire, lisible, communicable sous des formes diverses. Même le terme de "mémoire» ne convient pas. Cela est suffisamment ambitieux, et la réalisation peut se limiter à des performances modestes. Rappelons les sages préceptes du législateur : «il est important de rappeler que l'un des objectifs des TPE est d'aboutir à une réalisation concrète finale qui ne doit pas être réduite à la seule forme du "dossier", et encore moins de "mini-mémoire" alors que de multiples supports de production peuvent être envisagés. De même, il faut souligner que les productions issues des TPE doivent correspondre à un niveau raisonnable d'exigence, adapté aux compétences attendues d'élèves de première et de terminale ${ }^{2}$. Une classe de première, aussi brillante soit-elle, n'est pas l'antichambre du CNRS.

Cela implique que le professeur puisse guider et encadrer le travail des élèves. On ne laisse pas vagabonder un élève de première dans l'énorme masse de documentation qui est à sa disposition (au CDI, en bibliothèque, sur Internet, et parfois chez lui, avec toutes les disparités que cela peut entraîner) sans lui donner quelques conseils et consignes, sans quoi il perdra vite son temps. Le récent rapport de l'Inspection générale le signalait clairement : « une organisation trop libre présente plusieurs risques : laisser les élèves s'aventurer sur des sujets difficiles à maîtriser, qui finalement s'avèrent irréalistes ou au moins beaucoup plus pauvres qu'ils ne pouvaient le laisser espérer; laisser les élèves choisir des sujets sans lien même lointain avec les programmes, ce qui au bout de quelques mois engendre un fort sentiment d'insatisfaction chez les 
élèves en leur donnant l'impression qu'ils ont travaillé «pour rien» (p. 4)» ${ }^{3}$. L'inverse ne sera pas meilleur. Une trop grande contrainte dans l'organisation risque de tuer tout intérêt et finalement tout plaisir, aussi bien celui de se perdre par moment dans les méandres de l'information recueillie que celui de découvrir les réponses.

Cela, certes, ne va pas sans de réelles difficultés. On peut en retenir quelques-unes, pour mieux comprendre la portée du projet et les difficultés de sa mise en œuvre.

Bien des questions sont posées par des enseignants qui, par scrupule, vont consacrer beaucoup plus de temps aux TPE qu'il n'est raisonnablement possible. Il faut qu'aussi bien le professeur que l'élève apprennent à gérer leur temps. Dans ce genre d'activité, le mieux est toujours l'ennemi du bien. Il n'est pas opportun d'étaler sur toute l'année le travail, qui doit aboutir à un exposé et à un entretien. Entendre dire par des élèves que les TPE, « c'est un exposé qui dure toute l'année ", c'est entendre aussi un constat d'échec. "On passe d'une situation où l'ensemble du travail de l'élève se fait à l'échelle de la semaine à une activité qu'il s'agit de programmer sur plusieurs mois. On remarque une certaine lassitude, en particulier chez les élèves ayant commencé en octobre. Passer de la recherche documentaire à l'analyse et à la synthèse est un exercice ardu $[\ldots]$. La durée optimale à consacrer aux TPE semble être d'une quinzaine de semaines $»{ }^{4}$

La formulation des thèmes et des sujets est donc une phase de la mise en place à laquelle il faut prêter grande attention. Il ne faudrait pas oublier que les TPE doivent s'inscrire dans les programmes et qu'ils traitent, à leur manière, un des points du programme. Fallait-il publier une liste nationale des thèmes ? La remarque qui précède suffirait sans doute à définir le contenu des TPE. Si la liste proposée a une utilité, c'est surtout de " limiter le renouvellement annuel des thèmes et favoriser l'homogénéité et la continuité des TPE sur l'ensemble du cycle $" 5$.

Autre question délicate, celle de l'évaluation, qui est pour le moment le point faible des TPE. Il est très difficile d'aller contre des mentalités qui ne reconnaissent de valeur qu'à ce qui est évalué, c'est-à-dire noté et intégré, d'une manière ou d'une autre, à l'examen. Il est pourtant clairement dit que «les travaux personnels encadrés contribuent ainsi à l'appropriation des contenus des programmes d'enseignement et, par les compétences qu'ils développent chez les élèves, à la préparation du baccalauréat et à la poursuite d'études supérieures " ${ }^{6}$, il n'en reste pas moins vrai que des réticences importantes se font jour sur cette question de l'évaluation. Les hésitations de l'institution à ce sujet

3. Les travaux personnels encadrés, Rapport de l'IGEN (co-rapporteurs Jean Bottin, Marc Fort, Marc Baconnet).

4. Rapport de l'IGEN, p. 6.

5. B.O. $\mathrm{N}^{\circ} 24$ du 14-06-01 p. 276.

6. B.O. No 24 du 24-06-01 p. 1269). 
pour la classe de terminale ( à titre transitoire et pour la seule année scolaire 2001-2002, les élèves pourront choisir ou non de s'engager dans un TPE et de présenter ou non le travail effectué comme épreuve supplémentaire au baccalauréat ») laissent planer un sérieux doute sur l'avenir. Les TPE, uniquement à cause de la difficulté qu'il y a à les intégrer au baccalauréat (oserait-on suggérer qu'on pourrait les intégrer sans pour autant "passer » l'épreuve de TPE comme on passe d'autres épreuves?), iront-ils rejoindre le cimetière des innovations dont les ambitions furent grandes et la vie très courte?

De l'indifférence hostile au zèle intempestif, bien des attitudes peuvent freiner la réussite de l'innovation. Mais quels que soient son évolution et les nécessaires réajustements au fil des ans, on ne pourra faire l'économie d'un apprentissage de ce type. Il sera d'autant plus efficace que les enseignants sauront travailler de plus en plus entre eux et s'en tiendront à des réalisations concrètes, aux supports et présentations variés, en faisant preuve de modestie dans une entreprise dont le projet se veut, à juste titre, ambitieux. 